\title{
Status quo der Senioren- und Pflegeplanung und Handlungsempfehlungen für Kommunen
}

Grit Braeseke, Gerhard Naegele und Freja Engelmann

Inhaltsverzeichnis

14.1 Regelungen zur Pflegeplanung in den Bundesländern - 196

14.2 Handlungsfelder und Indikatoren der Pflegeplanung - 198

14.2.1 Handlungsfelder von Landespflegeplänen - 198

14.2.2 Handlungsfelder kommunaler Pflegeplanungen - 199

14.2.3 Indikatoren - 202

14.3 Handlungsempfehlungen für die kommunale Seniorenund Pflegeplanung - 202

$14.4 \quad$ Fazit -207

$$
\text { Literatur - } 207
$$




\section{- Zusammenfassung}

Die im § 9 SGB XI bundesgesetzlich vorgegebene Verantwortung der Bundesländer für die Vorhaltung einer leistungsfähigen pflegerischen Versorgungsstruktur wird in den Ländern unterschiedlich in die Praxis umgesetzt. Zum einen erfolgt die Pflegeplanung auf unterschiedlichen Ebenen (teilweise auf Länderund teilweise auf Kreisebene). Zum anderen variieren die Planungsinhalte und-verfahren stark. Nach einer kurzen Übersicht zu den gesetzlichen Regelungen der Bundesländer wird das Spektrum der Handlungsfelder von Pflegeplanungen auf Länder- und Kreisebene beschrieben. Anhand von vier konkreten Planungskonzepten werden anschließend die genutzten Indikatoren vorgestellt. Abschließend finden sich Handlungsempfehlungen zum konkreten Vorgehen bei der Senioren- und Pflegeplanung für die Stadt- und Landkreise.

According to § 9 Social Code (SGB) XI, the German states (Länder) are responsible for capacity-planning for the provision of efficient long-term care (LTC) services. Some states establish a state-wide LTC program, others have delegated LTC capacity-planning to the county level. The contents and procedures used in LTC planning vary substantially throughout the country. This article gives a brief overview on the distribution of responsibilities for LTC planning as well as the different frameworks and indicators used at different levels (state and county). Finally, policy recommendations for the LTC planning process on the regional level are given.

\subsection{Regelungen zur Pflegeplanung in den Bundesländern}

Wie im vorangegangenen Kapitel erörtert, ergibt sich aus § 9 SGB XI die Verantwortung der Bundesländer, eine leistungsfähige pflegerische Versorgungsstruktur vorzuhalten. Die konkrete Ausgestaltung dieses Auftrages erfolgt auf Länderebene. Mit Ausnahme von Sachsen haben alle Bundesländer entsprechende Pflegegesetze sowie dazu gehörige Verordnungen und Richtlinien erlassen.

- Tab. 14.1 listet die Verteilung der Zuständigkeiten für die Pflegeplanung nach Bundesländern auf.

- Tab. 14.1 Zuständigkeiten für die Pflegeplanung und Steuerungsgremien nach Bundesländern (Quelle: IGES; Stand 2019)

\begin{tabular}{|c|c|c|}
\hline Bundesland & Länderebene & Stadt- und Landkreise \\
\hline Baden-Württemberg & Landespflegeausschuss & $\begin{array}{l}\text { Erstellen Kreispflegepläne zu Bestand, } \\
\text { Bedarf und erforderlichen Maßnahmen zur } \\
\text { Bedarfsdeckung } \\
\text { Kommunale Pflegekonferenzen können } \\
\text { eingerichtet werden }\end{array}$ \\
\hline Bayern & Landespflegeausschuss & $\begin{array}{l}\text { Hinwirkungsverpflichtung zur Bereit- } \\
\text { stellung bedarfsgerechter Strukturen, } \\
\text { Pflegebedarfsermittlung als Bestandteil } \\
\text { der integrativen, regionalen Seniorenpoliti- } \\
\text { schen Gesamtkonzepte }\end{array}$ \\
\hline Berlin & $\begin{array}{l}\text { Erstellt Landesrahmenplan } \\
\text { Landesseniorenvertretung und Landes- } \\
\text { seniorenbeirat }\end{array}$ & $\begin{array}{l}\text { Bezirkliche Seniorenvertretungen und } \\
\text { Seniorendelegiertenversammlungen }\end{array}$ \\
\hline Brandenburg & $\begin{array}{l}\text { Erstellt Seniorenpolitische Leitlinien } \\
\text { Landespflegeausschuss }\end{array}$ & \\
\hline
\end{tabular}


- Tab. 14.1 (Fortsetzung)

\begin{tabular}{|c|c|c|}
\hline Bundesland & Länderebene & Stadt- und Landkreise \\
\hline Bremen & $\begin{array}{l}\text { Erstellung eines Altenplans (liegt nur } \\
\text { für die Stadt Bremen vor) }\end{array}$ & \\
\hline Hamburg & $\begin{array}{l}\text { Erstellung Landesrahmenplan } \\
\text { Landespflegeausschuss } \\
\text { Landes-Seniorenbeirat }\end{array}$ & $\begin{array}{l}\text { Pflegekonferenzen in den Bezirken können } \\
\text { eingerichtet werden } \\
\text { Bezirks-Seniorenbeiräte }\end{array}$ \\
\hline Hessen & $\begin{array}{l}\text { Erstellt Rahmenplan } \\
\text { Landespflegeausschuss }\end{array}$ & $\begin{array}{l}\text { Erstellen Pflegebedarfsplan } \\
\text { Pflegekonferenzen }\end{array}$ \\
\hline Mecklenburg-Vorpommern & $\begin{array}{l}\text { Erstellt Landesplan aus Basis der kom- } \\
\text { munalen Planungen } \\
\text { Landespflegeausschuss } \\
\text { Landesseniorenbeirat }\end{array}$ & $\begin{array}{l}\text { Erstellen kommunale Pflegesozialpläne } \\
\text { Kreisseniorenbeiräte und örtliche Senio- } \\
\text { renbeiräte }\end{array}$ \\
\hline Niedersachsen & $\begin{array}{l}\text { Erstellt Landespflegebericht } \\
\text { Landespflegeausschuss }\end{array}$ & $\begin{array}{l}\text { Erstellen örtliche Pflegeberichte } \\
\text { Örtliche Pflegekonferenzen }\end{array}$ \\
\hline Nordrhein-Westfalen & $\begin{array}{l}\text { Erstellt Landesförderplan und Bericht } \\
\text { zur Lage der Älteren }\end{array}$ & $\begin{array}{l}\text { Erstellen örtliche Pflegeplanungen (alle } \\
\text { zwei Jahre) } \\
\text { Kommunale Konferenzen Alter und Pflege }\end{array}$ \\
\hline Rheinland-Pfalz & Landespflegeausschuss & $\begin{array}{l}\text { Erstellen Pflegestrukturpläne } \\
\text { Regionale Pflegekonferenzen }\end{array}$ \\
\hline Saarland & $\begin{array}{l}\text { Erstellt Landespflegeplan } \\
\text { Landespflegeausschuss }\end{array}$ & \\
\hline Sachsen & Landespflegeausschuss & \\
\hline Sachsen-Anhalt & $\begin{array}{l}\text { Erstellt Pflegekonzeption des Landes } \\
\text { als Rahmenplan } \\
\text { Landespflegeausschuss und (seit Ende } \\
\text { 2017) sektorenübergreifender Landes- } \\
\text { pflegeausschuss }\end{array}$ & Erstellen Analyse der Pflegestrukturen \\
\hline Schleswig-Holstein & $\begin{array}{l}\text { Formuliert Zielsetzungen und Leitvor- } \\
\text { stellungen } \\
\text { Landespflegeausschuss }\end{array}$ & $\begin{array}{l}\text { Erstellung von Bedarfsplänen und regel- } \\
\text { mäßige Fortschreibung }\end{array}$ \\
\hline Thüringen & $\begin{array}{l}\text { Verantwortlich für Planung der } \\
\text { (teil)stationären Versorgungsstruktur } \\
\text { Erstellt Seniorenbericht } \\
\text { Landesseniorenrat }\end{array}$ & $\begin{array}{l}\text { Verantwortlich für Planung der ambulan- } \\
\text { ten Versorgungsstruktur } \\
\text { Kommunale Seniorenbeiräte und Senio- } \\
\text { renbeauftragter }\end{array}$ \\
\hline
\end{tabular}

Pflege-Report 2021

Die Anpassung der Landespflegegesetze an die Weiterentwicklung der Pflegegesetzgebung auf Bundesebene, ggf. der Auftrag der Kommunen zur Umsetzung der Planungsverpflichtung sowie der Umfang von Pflegeplanungen bezogen auf Handlungsfelder pflegerischer Versorgung variieren jedoch stark. Im folgen- den Abschn. 14.2 wird das inhaltliche Spektrum der Pflegeplanungen (Handlungsfelder und Indikatoren) vorgestellt. A Abschn. 14.3 gibt konkrete Empfehlungen für die Pflegeplanung auf Ebene der Stadt- und Landkreise und - Abschn. 14.4 enthält ein kurzes Fazit. 


\subsection{Handlungsfelder und Indikatoren der Pflegeplanung}

Einerseits wurde die Planungs- und Steuerungskompetenz der Kommunen im Pflegebereich im Zuge der Einführung der Sozialen Pflegeversicherung 1995 und des teilweisen Übergangs des Sicherstellungsauftrags für die pflegerische Versorgung auf die Pflegekassen ( $\$ 12$ Abs. 1 SGB XI) deutlich eingeengt, andererseits gehören die gesundheitliche und pflegerische Versorgung der Bevölkerung mit zum sozialen Daseinsvorsorgeauftrag der Kommunen. Entsprechend forderte die Expertenkommission für den 7. Altenbericht: „Im Sinne einer, verörtlichten Sozialpolitik' ist den Kommunen größerer Einfluss bei der Ausgestaltung von Infrastrukturen der Daseinsvorsorge einzuräumen. Dies gilt auch in den Feldern, in denen insbesondere die Sozialversicherungen inzwischen eine starke und dominierende Rolle einnehmen: Im Gesundheitswesen und in der Langzeitpflege“. Dabei sollen ,zentrale Elemente der Infrastrukturentwicklung, der Planung, der sozialräumlichen Entwicklung und Steuerung von Hilfen auf kommunaler Ebene angesiedelt und sowohl kompetenzrechtlich und finanziell flankiert werden" (BMFSFJ 2016, S. 293 f.).

Nach Ansicht der Bund-Länder-Arbeitsgruppe zur Stärkung der Rolle der Kommunen in der Pflege sollte eine bedarfsgerechte Versorgung älterer und pflegebedürftiger Menschen folgendes umfassen (BMG 2015, S. 2):

- eine neutrale, effiziente und wohnortna-

he Beratung, die die erforderlichen Informationen vermittelt und durch ein gutes Fallmanagement zur Verhinderung, Minderung und Bewältigung von Pflegebedürftigkeit beiträgt,

- ein breites Spektrum an Wohnformen, um Wahlfreiheit bezüglich des altengerechten Wohnens zu sichern und dem Wunsch nach größtmöglicher Selbständigkeit Rechnung zu tragen,
- eine leistungsfähige, ortsnahe und aufeinander abgestimmte ambulante und stationäre pflegerische Versorgung sowie

- die Gestaltung des Sozialraums bzw. der Lebenswelt, in dem bzw. der bürgerschaftliches Engagement, Familie und Nachbarschaftshilfe von Fachkräften sinnvoll ergänzt werden.

Diese Versorgung kann - nach Auffassung der Arbeitsgruppe - nur im engen Zusammenwirken der Beteiligten (Länder, Kommune, Pflegekassen und Leistungserbringer) sichergestellt werden.

Angesichts dieser Anforderungen sowie der demographischen Herausforderungen wird deutlich, dass eine eng gefasste „Pflegeinfrastrukturplanung“, die sich auf Pflegeheimstandorte und Platzzahlen beschränkt, nicht ausreichend ist. In den folgenden Abschnitten wird gezeigt, welche Themen die Pflegeplanungen auf Landesebene ( $\triangleright$ Abschn. 14.2.1) und auf Kreisebene ( $\triangleright$ Abschn. 14.2.2) adressieren und welche Indikatoren ( $\triangleright$ Abschn. 14.2.3) genutzt werden.

\subsubsection{Handlungsfelder von Landespflegeplänen}

Die inhaltliche Ausrichtung von Pflegeplanungen wird maßgeblich in den Landespflegegesetzen bestimmt. Neben den Strukturen der pflegerischen Versorgung im engeren Sinn, insbesondere stationäre und teilstationäre Pflegeeinrichtungen, werden dort mehrfach auch folgende Themen genannt:

- Hilfen im Vor- und Umfeld von Pflege

- Pflegeberatung

- Unterstützung pflegender Angehöriger

- Ehrenamt und bürgerschaftliches Engagement

Während der hessische Rahmenplan aus dem Jahr 1996 lediglich Planungsgrundsätze und Richtwerte für stationäre Pflegeeinrichtungen vorgibt, beinhalten die in den letzten 
Jahren erstellten Landespflegepläne (Berlin, Hamburg, Mecklenburg-Vorpommern, Saarland und Sachsen-Anhalt) jeweils auch eine Pflegeberichterstattung der letzten drei bis fünf Jahre vor der Planung und bieten somit eine Möglichkeit, die Ausgangslage im Land und in den Regionen (Kreise, Bezirke) umfassend zu bewerten.

Weiterhin ist festzustellen, dass die meisten Bundesländer über die Pflegeinfrastruktur im engen Sinne hinaus die ambulante Pflege sowie weitere Themen einbeziehen (mit Ausnahme des Saarlandes und Hessen):

- Berlin (2016): Arbeit, Ausbildung in der Altenpflege, Hospiz-/Palliativversorgung

- Hamburg (2015): Prognose des Pflegekräftebedarfs, Wohnformen, Information und Beratung, Demenz, niedrigschwellige Angebote, Altersarmut

- Mecklenburg-Vorpommern (2013): Fachkräftesicherung, Pflegeberatung, Prävention und Rehabilitation

- Sachsen-Anhalt (Altenhilfe und Pflege) (2008): Infrastruktur und Wohnen, Beteiligung und Teilhabe, Arbeitsmarkt, Altersarmut, Konsum Älterer

Baden-Württemberg hatte sich zunächst ebenfalls die Erstellung eines Pflegeplanes auf Landesebene vorgenommen. Diese Regelung wurde aber 2018 mit der umfassenden Novellierung des Landespflegegesetzes aufgehoben (Landespflegestrukturgesetz (LPSG)). Begründet wurde die Neuausrichtung vor allem mit einer bisher primär auf stationäre Einrichtungen ausgerichteten Förderung. Diese wurde als überholt betrachtet und als Konsequenz wird nun die Weiterentwicklung der Angebotsstruktur stärker auf den ambulanten Sektor ausgerichtet, im Sinne von ambulanten Wohnund Unterstützungsformen. Damit soll u. a. ein längeres Verbleiben der pflegebedürftigen Personen im häuslichen Umfeld gesichert werden. Betont wurde auch, dass diese Unterstützungsstrukturen für Menschen, die aufgrund ihres Alters, von Krankheit oder Behinderung auf Hilfe angewiesen sind, und für deren Angehörige deutlich umfassender sein müssen und in Form einer breiten Palette vielfältiger, miteinander vernetzter Angebote und Strukturen sozialraumbezogen und wohnortnah zu etablieren sind (Ministerium für Soziales und Integration Baden-Württemberg 2018, S. 2). In Konsequenz dieser Aussagen wurde die Aufgabe der Pflegeplanung den Stadt- und Landkreisen übertragen ( $\$ 4$ LPSG).

\subsubsection{Handlungsfelder kommunaler Pflegeplanungen}

Im Rahmen einer Studie im Auftrag des Hessischen Ministeriums für Soziales und Integration untersuchte das IGES Institut Anfang 2019 die Alten- und Pflegeplanung in den einzelnen Bundesländern (vgl. Braeseke et al. 2019). - Abb. 14.1 zeigt, welche Handlungsfelder in welcher Häufigkeit identifiziert wurden.

Die Handlungsfelder Gesundheit und Pflege sind Bestandteil aller betrachteten Alten-, Senioren- und Pflegekonzepte. Viele Pflegeplanungen berücksichtigen auch Aspekte der Gesundheitsversorgung, deren Qualität sich unmittelbar auf die pflegerische Versorgung auswirkt. Fast alle Konzepte beziehen auch ausdrücklich die Palliativversorgung mit ein (stationär in Hospizen oder ambulant durch spezialisierte Pflegedienste).

Die Einbeziehung von Ehrenamt und Bürgerschaftlichem Engagement sind weitere, fast in allen Konzepten enthaltene Handlungsfelder. Bereits auf der Ebene vieler Landespflegegesetze wird dies als Sollvorgabe genannt und auch im $\S 7 c$ SGB XI zur Errichtung der Pflegestützpunkte wird auf die Einbindung der Selbsthilfe sowie von Ehrenamt und bürgerschaftlichem Engagement hingewiesen. Dass dieser Aspekt als Handlungsfeld aufgegriffen wird, zeigt zum einen, dass Kommunen die Entwicklung einer aktiven Bürgergesellschaft vorantreiben, zum anderen trägt es sicher auch der Tatsache Rechnung, dass die Herausforderungen des demographischen 


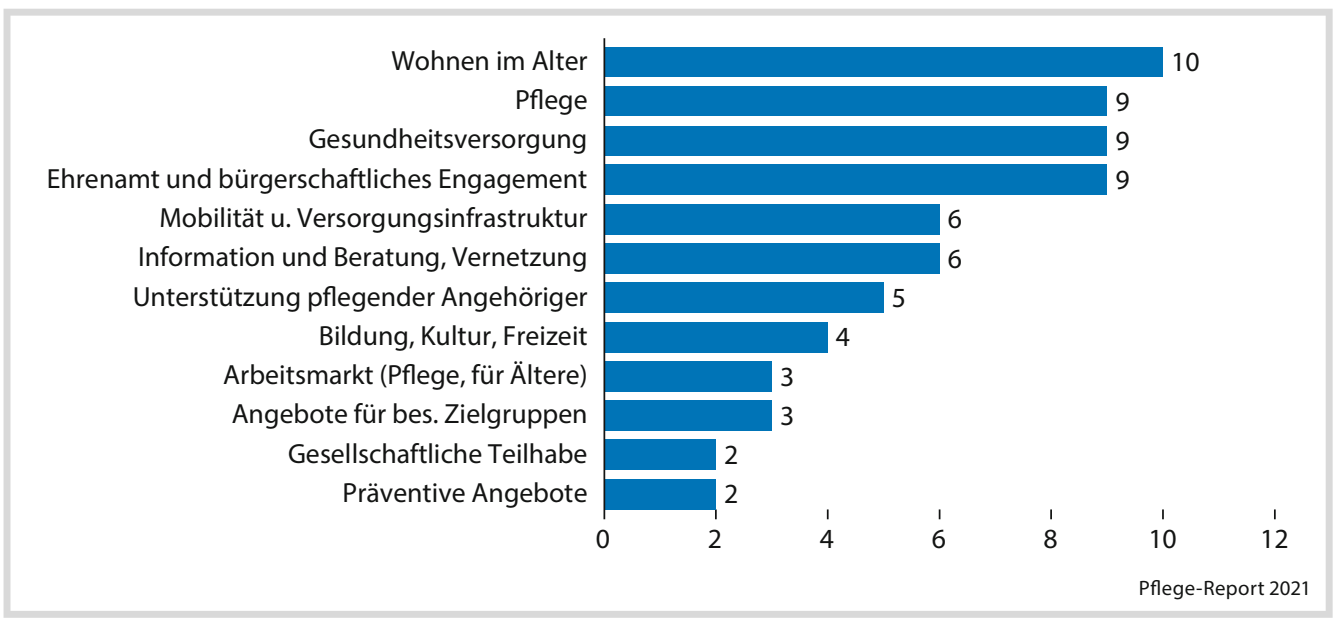

- Abb. 14.1 Art und Häufigkeit von Handlungsfeldern in Alten- und Pflegeplanungskonzepten $(n=10)($ Quelle: IGES)

Wandels nur unter Aktivierung aller Ressourcen zu bewältigen sein werden, einschließlich des sozialen Kapitals.

Das dritte wichtige Handlungsfeld umfasst das Wohnen im Alter, häufig auch ergänzt um die Aspekte Mobilität und Nahversorgung. Das zeigt die hohe Bedeutung dieses Themas im Alter - nur wenn ausreichend barrierearmer, seniorengerechter Wohnraum geschaffen werden kann, ist auch ein langer Verbleib der Älteren in der eigenen Wohnung möglich und damit die Umsetzung des Grundsatzes ,ambulant vor stationär“". Weiterhin ist dies ein Hinweis auf die kommunalen Einflussmöglichkeiten im Bereich des Wohnungsbaus.

An vierter Stelle steht das Handlungsfeld Information und Beratung sowie Vernetzung und Förderung sozialer Netzwerke. Im Rahmen der Pflegegesetze der letzten Jahre hat die Pflegeberatung einen besonderen Stellenwert erhalten. Vor allem die Entwicklung der Pflegestützpunkte oder lokaler Pflegeberatungsstellen ist hier zu nennen. Beim Aufbau der Beratungsinfrastruktur haben die Bundesländer jeweils verschiedene Akzente gesetzt, sodass Ratsuchende in Pflegefragen je nach Wohnort ein sehr unterschiedliches Beratungsangebot vorfinden. Die Kommunen haben im
Rahmen ihrer Daseinsfürsorge hier ein eigenständiges Betätigungsfeld und Gestaltungsmöglichkeiten und sollten diese Aufgabe nicht nur den Pflegekassen überlassen. Insofern hat das Pflegestärkungsgesetz III hier zu Recht eine wichtige Orientierung gegeben.

Ein besonderes Augenmerk sollte auf dem Thema Unterstützung pflegender Angehöriger liegen - nur jedes zweite der analysierten Konzepte berücksichtigt dieses Handlungsfeld explizit. Familienpflege ist eine der tragenden Säulen der Langzeitpflege in Deutschland und die Herausforderungen des Fachkräftemangels machen es erforderlich, diese Säule so gut wie möglich weiter zu stabilisieren - eine Aufgabe, die insbesondere Kommunen zukommt. Je schlechter dies gelingt, umso mehr pflegende Angehörige werden selbst zum Pflegefall.

Mit der bereits 2006 vorgenommenen gesetzlichen Verankerung der Seniorenpolitischen Gesamtkonzepte (SPGK) und dem 2010 vorgelegten Leitfaden zur Umsetzung war Bayern bundesweit Vorreiter bei der Einführung einer zukunftsorientierten kommunalen Seniorenpolitik. Die SPGK enthalten insgesamt elf Handlungsfelder und bilden damit alle für die pflegerische Versorgung relevanten Bereiche ab: 
1. Integrierte Orts- und Entwicklungsplanung: Bauleit- und Verkehrsplanung, barrierefreie/-arme Räume, Nahversorgungsinfrastruktur und Einzelhandel, öffentlicher Personennahverkehr

2. Wohnen zu Hause: Angebote von der Wohnberatung bis zu alternativen Wohnformen, besonders wichtig ist das ,Wohnen bleiben" einschl. Wohnraumanpassung und barrierefreies Bauen

3. Beratung, Information und Öffentlichkeitsarbeit: Soll generell den Zugang zu Versorgungseinrichtungen erleichtern - Informationsbroschüren, Anlauf- und Beratungsstellen etc.

4. Präventive Angebote: Setzt an vorhandenen Ressourcen und an der Selbstverantwortung für ein gesundes und aktives Altern an - sportliche Aktivitäten, Ernährungsberatung, Früherkennung (Hausärzte). Sturzprophylaxe und präventive Hausbesuche

5. Gesellschaftliche Teilhabe: Es sollen vorhandene Kontakte stabilisiert, neue begründet und damit der im Alter häufig zunehmenden Vereinsamung entgegengewirkt werden (Seniorenbeiräte, stadtteilorientierte Alten- und Servicezentren, Volkshochschulen, Mehrgenerationenhäuser etc.)

6. Bürgerschaftliches Engagement für und von Seniorinnen und Senioren: Förderung ehrenamtlichen Engagements (Pflegebegleiter, Ausbildungspaten, ,,Senioren ans Netz", Kinderbetreuung etc.)

7. Betreuung und Pflege: Beinhaltet neben den stationären Pflegeplätzen die häusliche Versorgung durch ambulante Dienste, Beratungsangebote wie Wohnberatung und Entlastungsangebote für pflegende Angehörige, Tagespflege, Kurzzeit- bzw. Verhinderungspflege, Betreutes Wohnen, Betreutes Wohnen zu Hause, ambulant betreute Wohngemeinschaften sowie unterschiedlichste niedrigschwellige Angebote und Betreuungsformen

8. Unterstützung pflegender Angehöriger: Fachstellen für pflegende Angehörige in jedem bayerischen Landkreis, niedrigschwellige Angebote, Betreuungsgruppen etc.

9. Angebote für besondere Zielgruppen: Menschen mit Demenz oder anderen psychischen Erkrankungen, vor allem auch mit Depressionen, Menschen mit Behinderung und Personen mit Migrationshintergrund

10. Kooperations- und Vernetzungsstrukturen: Träger- und ressortübergreifende Vernetzung, teilräumliche Arbeitsgemeinschaften, auch mit verschiedenen Trägerorganisationen, gemeinsame Fortbildungen

11. Hospiz- und Palliativversorgung: Sektorenübergreifende, regionale Netzwerke

Die Erarbeitung der SPGK verlief in den 96 Landkreisen und kreisfreien Städten Bayerns sehr unterschiedlich - sowohl zeitlich als auch inhaltlich. Das im Handlungsleitfaden 2010 formulierte Ziel, ,dass kommunale seniorenpolitische Gesamtkonzepte landesweit auf einem vergleichbaren und qualitätsgesicherten Niveau etabliert werden", konnte bis dato nicht erreicht werden. Auf Basis einer vom Kuratorium Deutsche Altershilfe erarbeiteten systematischen Bestandsanalyse zur Umsetzung der SPGK in Bayern wurde 2017 ergänzend eine Arbeitshilfe veröffentlicht, ,um auf lokaler Ebene den Aufbau ,sorgender Gemeinschaften" voranzutreiben" (StMAS 2017, S. 4). Da nach den praktischen Erfahrungen der Kommunen die inhaltlichen Aspekte der einzelnen Handlungsfelder nicht immer trennscharf sind und teilweise Querschnittsthemen umfassen, wurde in der Arbeitshilfe folgende Gliederung der Planung vorgeschlagen:

- Bereich Wohnen (Handlungsfelder Integrierte Orts- und Entwicklungsplanung sowie Wohnen zu Hause)

- Bereich Soziales (Handlungsfelder Präventive Angebote, Gesellschaftliche Teilhabe, Bürgerschaftliches Engagement und Angebote für besondere Zielgruppen)

- Bereich Pflege und Unterstützung (Handlungsfelder Betreuung und Pflege, Unterstützung pflegender Angehöriger, Hospizund Palliativversorgung). 
Die Handlungsfelder Beratung, Information und Öffentlichkeitsarbeit sowie Kooperationsund Vernetzungsstrukturen sollten als Querschnittsthemen in allen Bereichen berücksichtigt werden.

\subsubsection{Indikatoren}

Analog zur Vielfalt der Handlungsfelder werden in der Praxis auch vielfältige Indikatoren zur Operationalisierung herangezogen. Indikatoren sind Messgrößen, die die Bewertung bestimmter Sachverhalte ermöglichen. Sie können quantitativer (Maßzahl) oder qualitativer Art (Aussage) sein. Welche Indikatoren jeweils für welche Handlungsfelder geeignet sind, richtet sich in erster Linie nach dem Ziel der Planung. Die Analyse von Veränderungen bei den Indikatoren im Zeitverlauf unterstützt die Erfolgskontrolle.

Die Auswertung von einzelnen Pflegeplanungen hat gezeigt, dass sich die genutzten Indikatoren in drei Arten unterteilen lassen:

- Indikatoren zur Bevölkerungs-/Sozialstruktur und deren künftiger Entwicklung (Bevölkerung nach Altersgruppen, Bevölkerungsprognosen etc.)

- Bedarfsindikatoren, vor allem mit Blick auf Hilfe- und Pflegebedarf im Alter (Pflegebedürftige nach Alter, Pflegegrad und Art der Versorgung, Inanspruchnahme weiterer Leistungen)

- Bestandsindikatoren (differenzierte Versorgungsangebote in ambulanten, teil- und vollstationären Einrichtungen für unterschiedliche Bedarfslagen und Zielgruppen)

Eine Übersicht $\mathrm{zu}$ den in vier verschiedenen Planungskonzepten genutzten Indikatoren findet sich in Braeseke et al. 2019 (Tab. 4, S. 28 ff.). Empfehlungen für ein „Grundgerüst“ von Handlungsfeldern und Indikatoren für eine kommunale Senioren- und Pflegeplanung enthält der folgende $>$ Abschn. 14.3.

\subsection{Handlungsempfehlungen für die kommunale Senioren- und Pflegeplanung}

Allgemeine Planungsgrundsätze, die anhand der Erkenntnisse der Analysen empfohlen werden, sind:

- Möglichst eine integrierte Senioren- und Pflegeplanung umsetzen, auch Hilfen im Vor- und Umfeld von Pflege einbeziehen sowie präventive Ausrichtung (Vermeiden von Pflegebedürftigkeit, Gesundheitsförderung und Prävention).

- Die Planung sollte kleinräumig auf regionaler Ebene erfolgen (Kreis- und Gemeindeebene) und möglichst auch ggf. unterschiedlich geprägte Sozialräume berücksichtigen - die Vielfalt der Lebensverhältnisse ist sonst nicht abbildbar.

- „Kaskaden-Planung“ (von der Kreis- über die Gemeinde- bis zur lokalen Ebene der Wirkungskreise der Sozialwirtschaftsträger; Schubert 2019, S. 79) - nicht zu detailliert planen.

- Die Planung sollte zielgruppengenau erfolgen - welche besonderen Zielgruppen sind in der Region in den Blick zu nehmen (Menschen mit Demenz, pflegende Angehörige, jüngere Pflegebedürftige, Menschen mit Migrationshintergrund)?

- Die Einbeziehung der regionalen Akteure und der Bevölkerung in den Planungsprozess ist Voraussetzung für eine bedarfsgerechte Planung. Bedürfnisse und Bedarfe sind sehr individuell und ändern sich im Zeitablauf.

- Die Planungszyklen sollten zwischen drei und fünf Jahren liegen und der Planungshorizont zehn, höchstens 15 Jahre umfassen - längerfristige Planungen sind mit zu großen Unsicherheiten verbunden.

- Planung als kontinuierlichen Entwicklungsprozess gestalten und eine regelmäßige Altenberichterstattung (idealerweise jährlich) vornehmen. Das ermöglicht, (Fehl)Entwicklungen frühzeitig zu erken- 


\section{Die Roadmap für die integrierte Pflegesozialplanung}



- Abb. 14.2 Roadmap für die integrierte Pflegesozialplanung (Quelle: Roadmap für die integrierte Pflegesozialplanung aus Kraehmer 2016, S. 8)

nen und entsprechend rechtzeitig gegenzusteuern.

Inhaltliche Leitlinien für die Planung ergeben sich z. T. aus expliziten wie impliziten gesetzlichen Vorgaben (z. B. ambulant vor stationär, differenziertes Versorgungssystem, präventive Ausrichtung, Einbindung von Ehrenamt) und sollten um regionale Präferenzen oder Notwendigkeiten ergänzt werden (z. B. besonders hoher Stellenwert der Beratung in Pflegestützpunkten in Mecklenburg-Vorpommern, partizipatives Vorgehen, Rolle von besonderen Versorgungsangeboten in der Region, Migranten als Zielgruppe etc.).

Der Planungsablauf folgt im Wesentlichen dem bereits in den 1930er Jahren in den USA von Shewhart und Deming als kontinuierlicher Verbesserungsprozess entwickelten PDCA-Zyklus. Dieser besteht, nach einer Vorbereitungsphase, aus den vier Schritten Planen - Durchführen - Überprüfen - Anpassen/Handeln.

Die von Kraehmer et al. (2016) im Rahmen des Kompasses für die integrierte Pflegesozialplanung in Mecklenburg-Vorpommern entwickelte Roadmap ist ein gutes Beispiel für einen kompletten Planungszyklus mit einzelnen Planungsschritten nach diesem Schema (- Abb. 14.2). Sie enthält auch Hinweise auf für einzelne Schritte einzuplanende Zeiträume bei einem fünfjährigen Planungszyklus.

Besonders hervorgehoben wird in vielen Planungsbeispielen die Notwendigkeit der Bildung einer Steuerungsgruppe als lenkendes und steuerndes Organ. Sie bildet die Verbindung zwischen der Verwaltung und den unterschiedlichen, in den Planungsprozess einzubindenden Akteuren einschließlich der Bevölkerung.

Der Prozess der Planerstellung im engeren Sinne umfasst die Ermittlung von Handlungsbedarfen anhand einer Situationsanalyse. Ein gutes Vorgehen zeigt das folgende Schema des Planungsprozesses im Rhein-Kreis Neuss (inhaltlich allerdings auf den Pflegebedarf beschränkt). Sehr prägnant dargestellt ist parallel zu den Umsetzungsschritten auf der rechten Seite in Grau der beispielhafte Ablauf eines Beteiligungsprozesses (• Abb. 14.3).

Detaillierte Handlungsempfehlungen für eine Senioren- und Pflegeplanung entlang des Planungsprozesses auf Ebene der Stadt- und Landkreise enthält • Tab. 14.2. 


\section{Pflegebedarfsplanung}

Leistungsbild

\begin{tabular}{|c|c|c|}
\hline 01 & \multicolumn{2}{|c|}{ Untersuchung der Rahmenbedingungen } \\
\hline 02 & \multicolumn{2}{|c|}{ Bestandsaufnahme Angebot } \\
\hline & $\begin{array}{l}\text { Befragung Pflegeheime, } \\
\text { Tagespflegeeinrichtungen }\end{array}$ & $\begin{array}{c}\text { Befragung } \\
\text { ambulante Pflegedienste }\end{array}$ \\
\hline & $\begin{array}{l}\text { 3efragung der angrenzenden } \\
\text { Kreise bzw. kreisfreien Städte }\end{array}$ & $\begin{array}{l}\text { Erfassung der Planungen (inner- } \\
\text { halb und außerhalb des Kreises) }\end{array}$ \\
\hline \multicolumn{3}{|c|}{$\begin{array}{l}\text { Erfassung sonstiger } \\
\text { Pflegeinfrastrukturen }\end{array}$} \\
\hline 03 & \multicolumn{2}{|c|}{ Bedarfseinschätzung } \\
\hline
\end{tabular}

\section{Beteiligungsprozess}

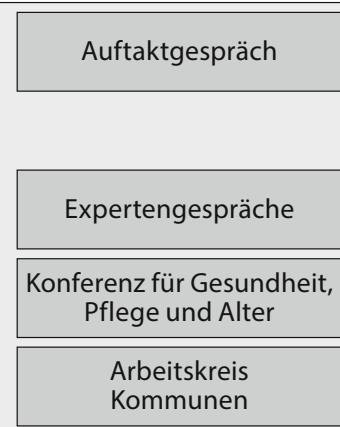

Bevölkerungsprognose

Pflegebedarfsprognose für drei Szenarien

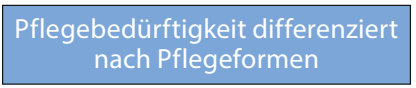

Pflegebedürftigkeit differenziert nach Pflegestufen alt und neu

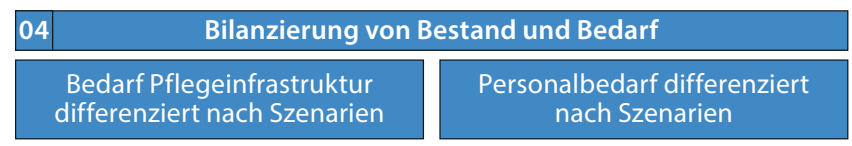

\section{5} Bewertung und Handlungsempfehlungen

Endbericht

Aufbau Monitoring - Fortschreibungskonzept

Fachkonferenz "örtliche Planung"

Konferenz für Gesundheit, Pflege und Alter

Ergebnispräsentation

- Abb. 14.3 Schema zum Planungsprozess im Rhein-Kreis Neuss (Quelle: ALP Institut 2017, S. 7)

- Tab. 14.2 Handlungsempfehlungen entlang des Planungsprozesses (Quelle: in Anlehnung an Braeseke et al. 2019, S. 66 ff.)

\section{Prozessschritte}

\section{Auftragsklärung}

\section{Handlungsoptionen/Indikatoren}

Planungsauftrag und Planungsverständnis klären (Leitbild formulieren, grundlegenden Ablauf klären)

Festlegung der verantwortlichen Person für die Planung und Koordination bzw. Bildung einer Steuerungsgruppe

Festlegung der einzubeziehenden Handlungsfelder und Identifizierung spezifisch zu berücksichtigender Zielgruppen 
- Tab. 14.2 (Fortsetzung)

\section{Prozessschritte}

2. Bevölkerungsanalysen

3. Bedarfseinschätzung

\section{Bestandsanalyse}

5. Bilanzierung, Ableitung von Zielen und Maßnahmen

\section{Handlungsoptionen/Indikatoren}

Bevölkerung nach Geschlecht und Altersgruppen

Bevölkerungsentwicklung nach Geschlecht und Altersgruppen (bis +10 bzw. 15 Jahre ausgehend vom Planungsjahr)

Haushaltsstrukturen, alleinlebende Ältere

Optional: Bevölkerung nach Staatsangehörigkeit (Migrationshintergrund), falls regional versorgungsrelevante Unterschiede auftreten

Pflegebedürftige (nach Alter, Geschlecht und Pflegegrad)

Pflegebedürftige Prognose (Zeithorizont analog Bevölkerungsprognose)

Personen mit Hilfe zur Pflege

Personen mit Demenz und ggf. weitere spezifische Zielgruppen

Niedrigschwelliger Hilfebedarf Älterer (Hauswirtschaft, Hilfen bei Besorgungen und Arzt-/Therapiebesuchen, Mobilitätshilfen)

Unterstützungsbedarf pflegender Angehöriger

Wohnsituation (insb. altersgerechte Wohnangebote)

Pflegestruktur: Einrichtungen (vollstationär, Kurzzeit-, Tages- und Nachtpflege, ambulante Dienste) und Personalausstattung

Prognose Pflegebedarf und Pflegepersonal bis 2035

Beratungsangebote

Angebote zur Unterstützung im Alltag

Haus- und fachärztliche Versorgung

Palliativ- und Hospizversorgung

Netzwerke

Bürgerschaftliches Engagement

\section{ÖPNV}

Nahversorgung, Apotheken

Bewertung der Angebote im Hinblick auf die identifizierten Bedarfe, Priorisieren

Abgleich mit den Alten- bzw. Pflegeplanungen benachbarter Regionen

Definition der Planungsziele (gewünschte Ergebnisse bzw. Wirkungen)

Was ist zu tun? Ableitung von Maßnahmen (Programme, Projekte)

Wie sind die Maßnahmen umzusetzen (Prozesse, Schritte, geeignete Partner)

Welche Ressourcen werden benötigt (Einsatz vorhandener Ressourcen, Investitionen etc.) 
Tab. 14.2 (Fortsetzung)

\begin{tabular}{|l|l|}
\hline Prozessschritte & Handlungsoptionen/Indikatoren \\
\hline $\begin{array}{l}\text { 6. Umsetzung der geplanten } \\
\text { Maßnahmen }\end{array}$ & $\begin{array}{l}\text { Dokumentation des Umsetzungsstandes, jährliche Bewertung der Ziel- } \\
\text { erreichung durch die Steuergruppe }\end{array}$ \\
\hline $\begin{array}{l}\text { Monitoring: Daten alle ein bis zwei Jahre neu erheben, kontinuierliche Alten- } \\
\text { berichterstattung implementieren }\end{array}$ \\
\hline kopplung & Prüfung der Wirksamkeit der Maßnahmen und der Bedarfsgerechtigkeit \\
\hline & $\begin{array}{l}\text { Erfolgskontrolle spätestens nach fünf Jahren, bei Fehlentwicklungen früher } \\
\text { gegensteuern, ggf. Anpassung der Ziele }\end{array}$ \\
\hline $\begin{array}{l}\text { Neustart des Planungsprozesses bei gravierenden Veränderungen der Ausgangs- } \\
\text { situation }\end{array}$ \\
\hline
\end{tabular}

Pflege-Report 2021

Wichtig ist es darauf hinzuwirken, dass die Planungen der Landkreise und kreisfreien Städte in einem Bundesland einer einheitlichen Grundstruktur folgen und grundlegende Indikatoren einheitlich erhoben bzw. berechnet werden. Das sichert die Vergleichbarkeit von einzelnen regionalen Planungen und ermöglicht das Zusammenführen auf Länderebene. Gleichzeitig wird der überregionale fachliche Austausch erleichtert.

In der Praxis hat sich gezeigt, dass eine Orientierungshilfe für Kommunen zur Umsetzung einer integrierten Senioren- und Pflegeplanung zielführend ist, auch um vergleichund auf Landesebene aggregierbare Ergebnisse zu erzielen. Unterstützung der Seniorenund Pflegeplanung in den Kommunen durch das Land wird in unterschiedlicher Form praktiziert:

\section{Erarbeitung von Leitlinien auf Länderebe-} ne:

- Bayern - Seniorenpolitische Leitlinien

- Brandenburg - Leitlinien der Seniorenpolitik

- Saarland - Rahmenempfehlung für kommunale seniorenpolitische Konzepte
Entwicklung von Handlungshilfen und -leitfäden für Kommunen im Auftrag des Landes

- Bayern - Leitfaden für kommunale Seniorenpolitik (StMAS 2010) und Arbeitshilfe zur Umsetzung seniorenpolitischer Gesamtkonzepte (StMAS 2017)

- Mecklenburg-Vorpommern - Kompass für die integrierte Pflegesozialplanung (Kraehmer et al. 2016)

- Nordrhein-Westfalen - Handbuch für Kommunen - Moderne Sozialplanung (Reichwein et al. 2011)

\section{Finanzielle Unterstützung der kommunalen Planungen}

- in Mecklenburg-Vorpommern

\section{Beratung der Kommunen durch vom Land finanzierte Stellen}

- Baden-Württemberg - Kommunalverband für Jugend und Soziales KVJS unterstützt die Kreise bei der Erstellung der Seniorenplanungen (Erhebung, Auswertung und Darstellung von Daten, Begleitung des Planungsprozesses, Ergebnisdokumentation und Berichterstellung)

- Brandenburg - Fachstelle Altern und Pflege im Quartier (FAPIQ) entstand im Rahmen der Pflegeoffensive 2015: im Bereich 
kommunale Altenhilfe- und Pflegeplanung bietet FAPIQ Beratung zu kommunalen Gestaltungsmöglichkeiten, zu Methoden und Instrumenten und Unterstützung bei lokalen Vernetzungsprozessen.

- Rheinland-Pfalz - Die Landeszentrale für Gesundheitsförderung in Rheinland-Pfalz e. V. (LZG) hat eine Servicestelle für kommunale Pflegestrukturplanung und Sozialraumentwicklung: Sie begleitet im Auftrag der Landesregierung Rheinland-Pfalz Kommunen bei der Pflegestrukturplanung und der Entwicklung einer kommunalen Infrastruktur für Senioren, behinderte Menschen, chronisch kranke sowie pflege- und unterstützungsbedürftige Menschen (LZG 2019).

- Thüringen - Programm zur Förderung von Sozialplanung in den Thüringer Kommunen (2015), Beratung der Kommunen durch das Institut für kommunale Planung und Entwicklung e. V.

Ein integriertes kommunales Steuerungssystem auf drei Ebenen (Case- und Care-Management sowie strategische Rahmenplanung), ein kommunales Pflegemarketing in enger $\mathrm{Ab}$ stimmung mit den Dienstleistern und der Aufbau eines Monitoringsystems erfordern entsprechend fachlich ausgebildetes Personal in den Verwaltungen. Hier kann das Land die Kreise über eine geeignete Aus- und Weiterbildungsförderung unterstützen.

\subsection{Fazit}

Die Senioren- und Pflegeplanung ist eine Thematik mit großer Vielfalt bundesweit. Auch innerhalb der Bundesländer finden sich teilweise sehr unterschiedliche Konzepte und Ausprägungen. Von der Pflege-, Altenhilfe-, Alten- bis zur integrierten Sozialplanung - das Spektrum reicht von einer engen Betrachtung (Pflegeinfrastruktur) bis zu einer ganzheitlichen und integrierten Berücksichtigung des Sozialraumes mit den Bereichen Gesundheit, Soziales, Integration, Inklusion, Jugend, Ar- beitsmarkt, Bildung, Verkehr, Stadtplanung, Stadtentwicklung und Umwelt.

Nicht zuletzt auch aufgrund des Fachkräftemangels in der Pflege geht es künftig nicht nur darum, die pflegerische Versorgung sicherzustellen, sondern den demographischen Wandel in den Kommunen aktiv zu gestalten: Senioren- und Pflegeplanung muss auch eine präventive Ausrichtung haben, um die Herausforderungen der demographischen Entwicklung in den Kommunen bewältigen $\mathrm{zu}$ können.

Die Notwendigkeit der verstärkten Abstimmung der Senioren- und Pflegeplanung mit den anderen Ressorts, um mit den begrenzten finanziellen Ressourcen die sozialpolitischen Ziele in einer Gesellschaft des Älterwerdens zu erreichen, findet aktuell im Modell einer integrierten und kooperativen Sozialplanung im Rahmen des Public Governance ihren Niederschlag (Schubert 2019, S. 56). Planung und Steuerung sozialer Dienstleistungen bleiben nicht länger nur Aufgaben der kommunalen Verwaltung, sondern es bilden sich Netzwerke der sozialen Verantwortung in den enger definierten Sozialräumen. „,Die Fragen der sozialen Planung für ältere Menschen werden in partizipativen institutionellen Arrangements verhandelt" (ebd.). Die Akteure entwickeln gemeinsam an runden Tischen, in Stadtteilkonferenzen oder Ideen-Camps tragfähige, räumlich spezifische Konzepte, die mittels örtlicher und regional mobilisierter Ressourcen umgesetzt werden. „Die Kommunen selbst können hier Koordinierungs-, Moderations- und Motivationsfunktionen übernehmen“ (Kruse 2019, S. 20).

\section{Literatur}

ALP Institut für Wohnen und Stadtentwicklung $\mathrm{GmbH}$ (2017) Pflegebedarfsplanung Rhein-Kreis Neuss

Bayerisches Staatsministerium für Arbeit und Sozialordnung, Familie und Frauen (2010) Kommunale Seniorenpolitik. Oktober 2010. StMAS, München

Bayerisches Staatsministerium für Arbeit und Soziales, Familie und Integration (2017) Seniorenpolitische Gesamtkonzepte. Erfahrungen und praktische Bei- 
spiele für die Umsetzung - eine Arbeitshilfe. März 2017. StMAS, München

Braeseke G, Naegele G, Engelmann F, Lingott N, Inkrot S (2019) Handlungsempfehlungen zur Altenhilfeplanung. Studie im Auftrag des Hessischen Ministeriums für Soziales und Integration. https:// soziales.hessen.de/sites/default/files/media/hsm/ handlungsempfehlungen_altenhilfeplanung_final_ 190513.pd. Zugegriffen: 30. Okt. 2020

Bundesministerium für Familie, Senioren, Frauen und Jugend (2016) Siebter Altenbericht. Sorge und Mitverantwortung in der Kommune - Aufbau und Sicherung zukunftsfähiger Gemeinschaften und Stellungnahme der Bundesregierung. BMFSFJ, Berlin

Bundesministerium für Gesundheit (2015) Empfehlungen der Bund-Länder-Arbeitsgruppe zur Stärkung der Rolle der Kommunen in der Pflege. BMG, Berlin

Kraehmer S (2016) Kompass für eine integrierte Pflegesozialplanung - Kurzfassung. Mai 2016. Hochschule Neubrandenburg

Kruse A (2019) Anforderungen der Gerontologie an die Planung für ältere Menschen. In: Schubert H (Hrsg) Integrierte Sozialplanung für die Versorgung im Alter: Grundlagen, Bausteine, Praxisbeispiele. Perspek- tiven Sozialwirtschaft und Sozialmanagement. Springer, Wiesbaden, S 19-41

Servicestelle für kommunale Pflegestrukturplanung und Sozialraumentwicklung bei der Landeszentrale für Gesundheitsförderung in Rheinland-Pfalz e. V. (2019) Arbeitshilfe zur Pflegestrukturplanung in RheinlandPfalz. LZG, Mainz

Ministerium für Soziales und Integration Baden-Württemberg (2018) Eckpunkte zur Novellierung des Gesetzes zur Umsetzung der Pflegeversicherung in BadenWürttemberg. Ministerium für Soziales und Integration Baden-Württemberg, Stuttgart

Reichwein A, Berg A, Glasen D, Junker A, Rottler-Nourbakhsch J, Vogel S, Trauth-Koschnick M (2011) Moderne Sozialplanung. Ein Handbuch für Kommunen. Kommunale Gemeinschaftsstelle für Verwaltungsmanagement (KGSt). Ministerium für Arbeit, Integration und Soziales des Landes Nordrhein-Westfalen, Düsseldorf

Schubert H (2019) Von der Altenhilfeplanung zur integrierten Sozialplanung im demografischen Wandel. In: Schubert H (Hrsg) Integrierte Sozialplanung für die Versorgung im Alter Grundlagen, Bausteine, Praxisbeispiele. Springer VS, Köln, S 43-74

Open Access Dieses Kapitel wird unter der Creative Commons Namensnennung 4.0 International Lizenz (http:// creativecommons.org/licenses/by/4.0/deed.de) veröffentlicht, welche die Nutzung, Vervielfältigung, Bearbeitung, Verbreitung und Wiedergabe in jeglichem Medium und Format erlaubt, sofern Sie den/die ursprünglichen Autor(en) und die Quelle ordnungsgemäß nennen, einen Link zur Creative Commons Lizenz beifügen und angeben, ob Änderungen vorgenommen wurden.

Die in diesem Kapitel enthaltenen Bilder und sonstiges Drittmaterial unterliegen ebenfalls der genannten Creative Commons Lizenz, sofern sich aus der Abbildungslegende nichts anderes ergibt. Sofern das betreffende Material nicht unter der genannten Creative Commons Lizenz steht und die betreffende Handlung nicht nach gesetzlichen Vorschriften erlaubt ist, ist für die oben aufgeführten Weiterverwendungen des Materials die Einwilligung des jeweiligen Rechteinhabers einzuholen.

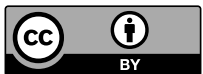

